\title{
Steric effects of ions in the charge-related wetting phenomena
}

\author{
C. K. Hua and I. S. Kang* \\ Department of Chemical Engineering, POSTECH, San 31, Hyoja-dong, Nam-gu, Pohang 790-784, South Korea \\ K. H. Kang \\ Department of Mechanical Engineering, POSTECH, San 31, Hyoja-dong, Nam-gu, Pohang 790-784, South Korea \\ H. A. Stone \\ School of Engineering and Applied Sciences, Harvard University, Cambridge, Massachusetts 02138, USA
}

(Received 2 July 2009; published 16 March 2010)

\begin{abstract}
Steric effects of ions on the charge-related wetting phenomena are studied. Along with a general treatment, three specific problems in two-dimensional system are considered: a droplet on an electrode, a droplet on a charged surface, and an electrowetting phenomenon on a dielectric. For computation of wetting tension, the electromechanical approach is adopted with the principle of mechanical force balance for each phase. The modified Poisson-Boltzmann equation, which was originally proposed by Bikerman [Philos. Mag. 33, 384 (1942)], is adopted for the analysis of the steric effects. It is found that the steric hindrance reduces significantly both the osmotic pressure and the electrical stress near the triple contact line. This reduction results in a considerable decrease in the wetting tension when the ratio of the capacitance per unit area of the electrical double layer to that of the dielectric layer is small.
\end{abstract}

DOI: 10.1103/PhysRevE.81.036314

PACS number(s): 47.55.np, 47.57.jd, 47.55.dr

\section{INTRODUCTION}

In the present work, we are concerned with the steric effects of ions in the charge-related wetting phenomena such as electrocapillarity and electrowetting on a dielectric. When an electrode is immersed in an electrolyte solution, the counterions are attracted to the electrode. The attracted ions are like ions and they push each other to exhibit collectively the electrocapillarity phenomenon. When we apply an electric field between the electrode immersed in an electrolyte droplet on a dielectric layer and the bottom electrode, we have a similar phenomenon which is called electrowetting. Under such configurations, if the electric field is strong enough, concentration of the counterions becomes very high near the electrolyte-dielectric interface and the steric effects are needed to be considered.

For microfluids handling, electrowetting has been regarded as a very efficient tool and it has been applied to many problems such as a lab on a chip and a liquid lens (see the review paper [1]). Recently it finds even more promising applications by the technique of low-voltage electrowetting, in which a high quality thin dielectric layer is used [2-5]. In such systems, it becomes easier to get stronger electric field to attract more counterions toward the opposite electrode. The ions may be very concentrated and are to experience some steric effects. Thus far, however, the steric effects of ions on the wetting phenomenon have not been analyzed.

The steric effects in the electrolyte system have long been studied and there are many references (see Kilic et al. [6] and the references therein). In the present work, we do not intend to develop any new theory on the steric effects in the elec-

\footnotetext{
*iskang@postech.ac.kr
}

trolyte system. We rather adopt a specific theory available in the literature to show how the steric effect theory is incorporated into the general setting for computation of wetting tension at the triple point of a contact line. So, if a better theory is developed for the steric effects in an electrolyte system, the old theory may be replaced by the new one.

In the present work, we adopt the theory based on the modified Poisson-Boltzmann (mPB) equation. The theory was originally proposed by Bikerman [7] and has been adopted for various analyses of electrolyte systems $[6,8-10]$ and ionic liquid systems [11]. In fact, Bickerman's model has a long and interesting history and has been rediscovered by several researchers (see the review paper by Bazant et al. [12]). The model is concise and has a nice property of allowing analytical approaches to application problems. As will be shown later, the $\mathrm{mPB}$ equation enables us to also do analytical studies for this work.

The electromechanical approach is adopted for the present study of charge-related wetting phenomena. In the approach, the electrical effect can be separated out from surface tension and obtained by integrating the Maxwell stress with the osmotic-pressure term along the drop surface. The electrowetting problems of conducting fluids have been studied by the approach $[13,14]$. It was adopted also for the study of wetting phenomena of the electrolyte systems, in which the steric effects of ions have not been considered [15]. Thus, the present study may be regarded as an extension of the previous work to include the steric effects. To see the steric effects on the wetting phenomena concretely, three most typical subproblems in two-dimensional (2D) system are considered as in the previous work: (i) a droplet on a constant potential substrate surface, (ii) a droplet on a constant charge substrate surface, and (iii) an electrowetting of a droplet on a dielectric layer. 


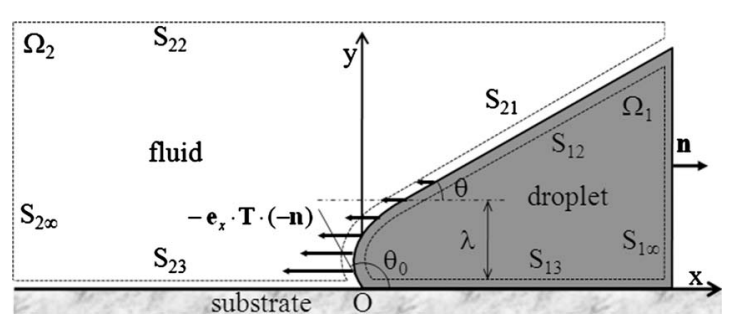

FIG. 1. Coordinate systems and definition of variables.

\section{PRELIMINARY NOTIONS}

\section{A. Wetting tension}

In the present work, we are interested in the case where the electrical effect generates an extra stress at the asymptotically thin layer near the solid surface. It is assumed that the stress shows an integrable singularity behavior as the thickness goes to zero. Because of this stress, the fluid-fluid interface (the interface 1-2) is deformed to produce a macroscopic contact angle change as sketched in Fig. 1. For this situation, we want to express the wetting tension in terms of the electrical stress and the osmotic pressure. We consider a region near the triple contact line, which can be enlarged and approximated by a 2D system as shown in Fig. 1. In Figs. 1-3 denote the phases and $\gamma_{i j}$ is the interfacial tension of interface of the phases $i$ and $j$ when there is no electrostatic effect. For later use, $S_{i j}$ is introduced. $S_{i j}$ is the surface of the $i$ th phase facing the phase $j$. The arclength along the interface measured from the triple point is denoted by $s$. For each phase boundary, the outward normal vector $\mathbf{n}$ is defined. The thickness of a microscopically thin layer inside the drop phase near the solid surface, in which the electric field is concentrated, is denoted by $\lambda$ and we consider the limiting case $\lambda \rightarrow 0$. The intrinsic contact angle right at the contact point is denoted by $\theta_{0}$, while the constant contact angle outside the thin layer is denoted by $\theta$. Generally the (macroscopic) contact angle refers to $\theta$.

In the following theories of charge-related wetting phenomena, we assume that the interfacial tensions themselves are not changed due to the change in electrostatic situation.

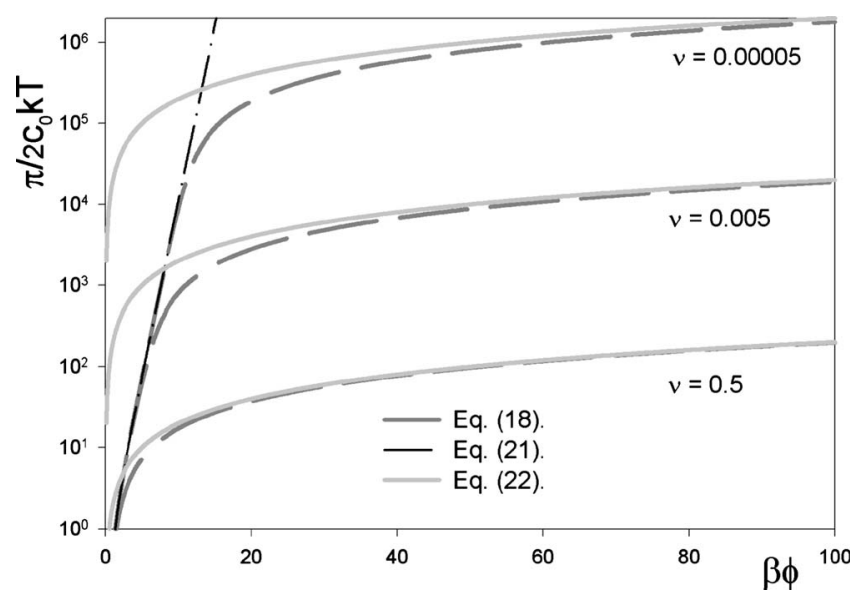

FIG. 2. The dimensionless osmotic pressure $\left(\pi / 2 c_{0} k T\right)$ vs the dimensionless potential $(\beta \phi)$.

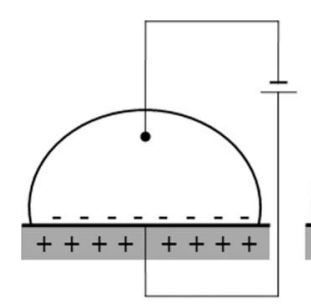

(a)

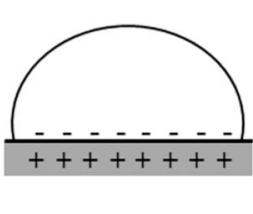

(b)

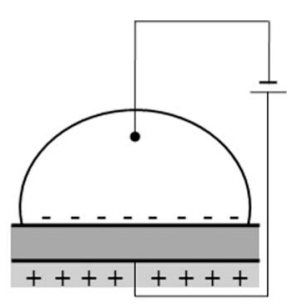

(c)
FIG. 3. Three most common charge-related wetting phenomena.

Of course, the changes in charge distributions may cause some intrinsic changes in molecular structures at the interfaces. However, those effects are neglected at the present level of accuracy of analysis. In the more sophisticated work, those points may be included.

Now, if we define the wetting tension $W_{e l}$ as the negative $x$-directional force per unit depth due to electrical effect, exerted on the asymptotically short portion of the interface 1-2, the (macroscopic) balance between the negative and positive $x$-directional forces gives

$$
W_{e l}+\gamma_{23}=\gamma_{13}+\gamma_{12} \cos \theta
$$

The wetting tension can be computed in terms of the stress tensor as

$$
W_{e l}=-\mathbf{e}_{x} \cdot \int_{S_{12}+S_{21}} \mathbf{T} \cdot(-\mathbf{n}) d s
$$

where $d s$ is the differential arclength along the interface starting from the triple point and $\mathbf{n}$ is the outward unit normal from the surface $S_{12}$ or $S_{21}$. In Eq. (2), the (total) stress tensor $\mathbf{T}$ has two contributions of the osmotic pressure and electrical stress as

$$
\mathbf{T}=-\pi \mathbf{I}+\mathbf{T}^{e}=-\pi \mathbf{I}+\epsilon\left[\mathbf{E} \mathbf{E}-\frac{1}{2} E^{2} \mathbf{I}\right]
$$

where $\pi$ is the osmotic pressure and $\mathbf{T}^{e}$ is the electrical Maxwell stress tensor. As mentioned earlier, $\mathbf{T}$ becomes negligible outside the region of thickness $\lambda$ [no contribution to the integration of Eq. (2)].

It must be pointed out again that, in the present theory, the interfacial tensions in Eq. (1) are assumed to be constant with respect to the change of electric field. Another point is that the expression of the (total) stress tensor in terms of osmotic pressure and the electrical stress is valid when there is no fluid motion, i.e., in a static situation. However, the formula can also be valid for the dynamic situations under the condition that $\lambda \rightarrow 0$. This point will be discussed in detail later.

When there is no electrical effect, the interface 1-2 must be straight and we have

$$
\gamma_{23}=\gamma_{13}+\gamma_{12} \cos \theta_{0}
$$

From Eqs. (1) and (4), we can derive the famous equation for electrowetting, 


$$
\cos \theta=\cos \theta_{0}+\frac{W_{e l}}{\gamma_{12}} .
$$

Now we want to show that, under the assumptions mentioned above, while the macroscopic contact angle $\theta$ is changed due to the electrical effects, the intrinsic contact angle $\theta_{0}$ (the contact angle right at the triple point) is not changed. For the magnified portion of $S_{12}$ of the thickness of order $\lambda$, the interface is deformed according to the stress balance condition

$$
[\mathbf{T} \cdot(-\mathbf{n})]_{S_{12}+S_{21}}=\gamma_{12} \mathbf{n}_{1}\left(\frac{1}{R_{1}}\right),
$$

where $\mathbf{n}_{1}$ denotes the outward normal at the surface $S_{12}$ and $R_{1}$ is the radius of curvature. The negative $x$-directional component of Eq. (6) is

$$
-\mathbf{e}_{x} \cdot[\mathbf{T} \cdot(-\mathbf{n})]_{S_{12}+S_{21}}=\gamma_{12} \sin \theta\left(-\frac{d \theta}{d s}\right) .
$$

By integrating the above equation under the assumption that the interfacial tension is not changed, we have

$$
\begin{aligned}
W_{e l} & =\gamma_{12} \int_{0}^{\infty} \sin \theta\left(-\frac{d \theta}{d s}\right) d s \\
& =\gamma_{12} \int_{\theta_{0}}^{\theta_{\infty}}(-\sin \theta) d \theta \\
& =\gamma_{12}\left(\cos \theta-\cos \theta_{0}\right) .
\end{aligned}
$$

In the above, we use the fact that $\theta_{\infty}=\theta$. Equation (7) is identical to Eq. (5) and this proves our assertion. Here, it should be mentioned that the same conclusion was drawn earlier by numerical computation of Buehrle et al. [16].

As we have seen above, all we need to do for computing the wetting tension is to evaluate the osmotic pressure and the electrical stress and then integrate the total stress along the contour starting from the triple point.

\section{B. Osmotic pressure in terms of E-field}

For any closed surface $\Sigma$ in one phase (say, phase 1), the mechanical force balance can be stated as [17]

$$
\int_{\Sigma} \mathbf{n} \cdot \mathbf{T} d \Sigma=\int_{\Sigma} \mathbf{n} \cdot\left[-\left(\pi+\frac{1}{2} \epsilon E^{2}\right) \mathbf{I}+\epsilon \mathbf{E E}\right] d \Sigma=0 .
$$

As mentioned earlier, this relation is valid under the static condition. For the dynamic situation, this relation is valid only under certain limiting conditions. The discussion about this will be given in Sec. II C.

For the same closed surface $\Sigma$, we can now show the following relation by using the Maxwell equations and the vector identities:

$$
\int_{\Sigma} \epsilon(\mathbf{n} \cdot \mathbf{E E}) d \Sigma=\int_{\Omega}\left[\rho_{f} \mathbf{E}+\frac{\boldsymbol{\epsilon}}{2} \nabla E^{2}\right] d \Omega
$$

where $\Omega$ is the volume closed by $\Sigma$ and $\rho_{f}$ is the free charge density that satisfies $\epsilon \boldsymbol{\nabla} \cdot \mathbf{E}=\rho_{f}$. By substituting Eq. (9) into
Eq. (8) and using the divergence theorem, we have

$$
\nabla \pi=\rho_{f} \mathbf{E} .
$$

The physical meaning of the above equation is that the electrical force on a certain part of the fluid via exerting force on the free charges must be balanced by the osmotic-pressure gradient. The same formula is shown also in an earlier work [18].

\section{Mechanical force balance}

As mentioned earlier, mechanical force balance (8) is valid for the static cases. The general equation of motion for a Newtonian fluid under electric field is

$$
\rho\left[\frac{\partial \mathbf{u}}{\partial t}+\mathbf{u} \cdot \boldsymbol{\nabla} \mathbf{u}\right]=\boldsymbol{\nabla} \cdot \mathbf{T} ; \quad \boldsymbol{\nabla} \cdot \mathbf{T}=-\boldsymbol{\nabla} p+\boldsymbol{\nabla} \cdot \boldsymbol{\sigma}^{h}+\boldsymbol{\nabla} \cdot \mathbf{T}^{e}
$$

where $\mathbf{u}$ is the velocity vector and $\tau^{h}$ is the viscous stress tensor given by

$$
\tau^{h}=\mu\left[\nabla \mathbf{u}+\nabla \mathbf{u}^{T}\right]
$$

After introducing appropriate characteristic scales, we nondimensionalize the equation as

$$
\begin{aligned}
\frac{\rho u_{c}^{2}}{\epsilon E_{c}^{2}} \frac{l_{c}^{e}}{l_{c}^{h}}\left[\frac{\partial \mathbf{u}^{*}}{\partial t^{*}}+\mathbf{u}^{*} \cdot \nabla^{*} \mathbf{u}^{*}\right] \\
\quad=-\frac{p_{c}}{\epsilon E_{c}^{2}} \frac{l_{c}^{e}}{l_{c}^{p}} \nabla^{*} p^{*}+\frac{\left(\mu u_{c} / l_{c}^{h}\right)}{\epsilon E_{c}^{2}} \frac{l_{c}^{e}}{l_{c}^{h}} \boldsymbol{\nabla}^{*} \cdot \boldsymbol{\tau}^{* h}+\boldsymbol{\nabla}^{*} \cdot \mathbf{T}^{* e}
\end{aligned}
$$

where $l_{c}^{h}, l_{c}^{p}$, and $l_{c}^{e}$ are the length scales for the flow field, pressure field, and the electric field, respectively. As mentioned before, we assume that the electric field is confined in a thin layer of thickness $\lambda$. So, for the electric-field length scale $l_{c}^{e}=\lambda$ is a good choice. If the assumptions,

$$
\frac{\rho u_{c}^{2}}{\epsilon E_{c}^{2}} \frac{l_{c}^{e}}{l_{c}^{h}} \ll 1, \quad \frac{\left(\mu u_{c} / l_{c}^{h}\right)}{\epsilon E_{c}^{2}} \frac{l_{c}^{e}}{l_{c}^{h}} \ll 1,
$$

are valid, the inertia and viscous terms can be neglected. Thus if we take the pressure scale as $p_{c}=\epsilon E_{c}^{2}$ and the length scale of pressure field as $l_{c}^{p}=l_{c}^{e}$, we have

$$
\boldsymbol{\nabla} \cdot \mathbf{T}=\boldsymbol{\nabla} \cdot\left[-p \mathbf{I}+\mathbf{T}^{e}\right]=\mathbf{0} .
$$

Here we should note that Eq. (14) is exactly the same as Eq. (8) if the pressure $p$ is the osmotic pressure $\pi$. From Eq. (13), we can see also that the assumptions are valid if $\lambda \rightarrow 0$ while the flow field has some finite velocity and length scales. Then the evaluation of the integral of wetting tension [Eq. (2)] can be easily achieved by using the well-known property of the stress tensor as in previous works [13-15],

$$
\int_{\Omega} \boldsymbol{\nabla} \cdot \mathbf{T} d \Omega=\int_{\Sigma} \mathbf{n} \cdot \mathbf{T} d \Sigma=\mathbf{0} .
$$




\section{ANALYSIS}

\section{A. Steric effects on the osmotic pressure}

In this section, we analyze the steric effects on the osmotic pressure by using the general result derived in Sec. II [Eq. (10)]. Although Eq. (10) can be applied to any steric effect model, we adopt a specific model proposed by Bikerman to derive some concrete results [7]. According to their model, the positive and negative charges have the modified Boltzmann distribution [6],

$$
c_{ \pm}=\frac{c_{0} e^{\mp \beta \phi}}{1+\nu(\cosh \beta \phi-1)}
$$

where $c_{0}$ is the bulk number density of each ion at zero potential and $\beta=z e / k T$. The parameter $\nu$ stands for the steric effect and defined as $\nu=2 a^{3} c_{0}$, where $a$ is the ionic radius (for simplicity, the same size is assumed for the positive and negative ions). Physically $\nu$ represents the volume fraction occupied by the ions at zero potential in the bulk $[6,9]$.

Now we have

$$
\rho_{f}=z e\left(c_{+}-c_{-}\right)=\frac{-2 c_{0} z e \sinh \beta \phi}{1+\nu(\cosh \beta \phi-1)} .
$$

By substituting Eq. (17) into Eq. (10) with $\mathbf{E}=-\nabla \phi$ and integrating the resulting equation with the boundary condition $\pi=0$ at $\phi=0$, we have the formula of the osmotic pressure that includes the steric effect

$$
\pi=2 c_{0} k T \frac{1}{\nu} \ln [1+\nu(\cosh \beta \phi-1)]
$$

It would be interesting to consider the limiting case of Eq. (18) for negligible steric effect, i.e., $0<\nu \ll 1$. In this case, Eq. (18) is reduced to

$$
\pi=2 c_{0} k T\left[\frac{c_{+}+c_{-}}{2 c_{0}}-1\right] .
$$

This is the same as the well-known result for dilute electrolyte solution derived by Marcus many years ago [19]. The first steric effect can be obtained from Eq. (18) as

$$
\pi=2 c_{0} k T\left[\frac{c_{+}+c_{-}}{2 c_{0}}-1\right]\left[1+\left(\frac{c_{+}+c_{-}}{2 c_{0}}+1\right) \frac{\nu}{2}+\cdots\right] .
$$

Now, let us pay attention to the limiting form of Eq. (18) for the case of high electric potential (i.e., $\phi \rightarrow \infty$ ). If there is no steric effect $(\nu=0)$, we have

$$
\pi=2 c_{0} k T[\cosh \beta \phi-1] \rightarrow c_{0} k T e^{\beta \phi} .
$$

On the other hand, for the cases of finite steric effect, Eq. (18) can be reduced to

$$
\pi \rightarrow 2 c_{0} k T\left(\frac{\beta \phi}{\nu}\right) .
$$

From Eqs. (21) and (22), we can see that, if there is no steric effect, the osmotic pressure increases exponentially in the high potential limit. On the other hand, under the finite steric effect, it increases linearly with the electric potential. The results of Eqs. (18), (21), and (22) are shown in Fig. 2.

\section{B. Steric effects on the wetting tension}

In this work, we consider the steric effect on the wetting tension of the charge-related wetting phenomena. As in Kang et al. [15], we consider the three cases shown in Fig. 3. The first case is that the substrate surface has the constant potential (case I). The second case is that the substrate surface has the constant charge (case II). Third we consider the case of electrowetting problem, i.e., the droplet on a dielectric layer (case III).

All cases shown above can be represented as shown Fig. 1. For such a system, the wetting tension can be computed by

$$
W_{e l}=-\mathbf{f} \cdot \mathbf{e}_{x} ; \quad \mathbf{f}=-\int_{S_{12}+S_{21}} \mathbf{T} \cdot \mathbf{n} d s .
$$

The osmotic pressure is given by Eq. (18) and the electric field for evaluation of the wetting tension can be computed by solving the modified Poisson-Boltzmann equation for each phase $j[6,9]$,

$$
\nabla^{2} \phi=\frac{\kappa^{2}}{\beta} \frac{\sinh \beta \phi}{1+\nu[\cosh \beta \phi-1]},
$$

where $\kappa^{2}=2 c_{0} z^{2} e^{2} / \epsilon k T$, with the boundary conditions

$$
\begin{gathered}
\phi(x, 0)=\phi_{0}(x), \quad \phi(x, \infty)=0, \\
\frac{\partial \phi}{\partial x}( \pm \infty, y)=0 .
\end{gathered}
$$

Now, let us consider the three cases separately below.

\section{Constant potential problem (case I)}

In this case, the potential is constant along the substrate surface [in Eq. (25)], $\phi_{0}(x)=\phi_{0}=$ constant). The net surface force per unit depth exerted on the interface of droplet and external fluid (the interface 1-2) can be computed very effectively by using the mechanical force balance mentioned in Sec. II

$$
\begin{aligned}
\mathbf{f}=\mathbf{f}_{1}+\mathbf{f}_{2} & =-\int_{S_{12}} \mathbf{T} \cdot \mathbf{n} d s-\int_{S_{21}} \mathbf{T} \cdot \mathbf{n} d s, \\
& =\int_{S_{1 \infty}+S_{13}} \mathbf{T} \cdot \mathbf{n} d s+\int_{S_{2 \infty}+S_{23}+S_{22}} \mathbf{T} \cdot \mathbf{n} d s .
\end{aligned}
$$

For $f_{1 x}^{(I)}=\mathbf{e}_{x} \cdot \mathbf{f}_{1}$, we have $E_{n}=\mathbf{n} \cdot \mathbf{E}=0$ along $S_{1 \infty}$ and $\mathbf{n} \cdot \mathbf{e}_{x}=0$ along $S_{13}$, so it can be easily shown that

$$
f_{1 x}^{(I)}=-\int_{S_{1 \infty}}\left[\pi+\frac{1}{2} \epsilon_{1} E^{2}\right] d s .
$$

The osmotic pressure is given in Eq. (18) and we need $E_{y}=\mathbf{e}_{y} \cdot \mathbf{E}=-\frac{\partial \phi}{\partial y}$ for $x \rightarrow \infty$. This can be obtained by integrating Eq. (24) with respect to $y$ after multiplying $\phi$ to both sides [6] and we have 


$$
\frac{1}{2} \epsilon_{1} E_{y}^{2}=\frac{2 c_{0} k T}{\nu_{1}} \ln \left[1+\nu_{1}(\cosh \beta \phi-1)\right] .
$$

Therefore, we have

$$
\begin{aligned}
f_{1 x}^{(I)} & =-\int_{0}^{\infty}\left[\pi+\frac{1}{2} \epsilon_{1} E_{y}^{2}\right]_{x=\infty} d y \\
& =-\int_{\phi(\infty, \infty)}^{\phi(\infty, 0)}\left[\frac{\pi+\frac{1}{2} \epsilon_{1} E_{y}^{2}}{E_{y}}\right] d \phi \\
& =-2 \sqrt{\epsilon_{1} c_{10} k T} \int_{0}^{\phi_{1 \infty}} \sqrt{\frac{1}{\nu_{1}} \ln \left[1+\nu_{1}(\cosh \beta \phi-1)\right]} d \phi \\
& =-2 \sqrt{2}\left[\frac{c_{10} k T}{\kappa_{1}}\right] \int_{0}^{\beta \phi_{1 \infty}} \sqrt{\frac{1}{\nu_{1}} \ln \left[1+\nu_{1}(\cosh \eta-1)\right]} d \eta
\end{aligned}
$$

where $\phi_{1 \infty} \equiv \phi(\infty, 0)$. In a similar way, we can show that with $\phi_{2 \infty} \equiv \phi(-\infty, 0)$,

$$
\begin{aligned}
f_{2 x}^{(I)} & =\int_{0}^{\infty}\left[\pi+\frac{1}{2} \epsilon_{2} E_{y}^{2}\right]_{x=-\infty} d y \\
& \left.=2 \sqrt{2}\left[\frac{c_{20} k T}{\kappa_{2}}\right] \int_{0}^{\beta \phi_{2 \infty}} \sqrt{\frac{1}{\nu_{2}} \ln \left[1+\nu_{2}(\cosh \eta-1)\right.}\right] d \eta .
\end{aligned}
$$

In the above equations, $\kappa_{j}=\left(2 c_{j 0} z^{2} e^{2} / \epsilon_{j} k T\right)^{1 / 2}$. Since $W_{e l}^{(I)}=-f_{1 x}^{(I)}-f_{2 x}^{(I)}$ and $\phi_{1 \infty}=\phi_{2 \infty}=$ constant $=V$, the wetting tension for the case of constant potential is given by

$$
\begin{aligned}
W_{e l}^{(I)=} & \left.2 \sqrt{2}\left[\frac{c_{10} k T}{\kappa_{1}}\right] \int_{0}^{\beta V} \sqrt{\frac{1}{\nu_{1}} \ln \left[1+\nu_{1}(\cosh \eta-1)\right.}\right] d \eta \\
& \left.-2 \sqrt{2}\left[\frac{c_{20} k T}{\kappa_{2}}\right] \int_{0}^{\beta V} \sqrt{\frac{1}{\nu_{2}} \ln \left[1+\nu_{2}(\cosh \eta-1)\right.}\right] d \eta .
\end{aligned}
$$

Now, let us consider some special cases of no steric effect $\left(\nu_{j} \rightarrow 0\right)$ and the first correction when the steric effect is small. When the steric effect is negligible, $\ln \left[1+\nu_{j}(\cosh \eta-1)\right] \simeq \nu_{j}(\cosh \eta-1)$, and we have

$$
\int_{0}^{\beta V} \sqrt{\frac{1}{\nu_{j}} \ln \left[1+\nu_{j}(\cosh \eta-1)\right]} d \eta=\frac{2 \sqrt{2}}{\beta}\left(\cosh \frac{\beta V}{2}-1\right),
$$

$$
j=1,2 .
$$

Therefore, we have the wetting tension formula without the steric effect

$$
W_{e l}^{(I)}=8 k T\left[\frac{c_{10}}{\kappa_{1}}-\frac{c_{20}}{\kappa_{2}}\right]\left[\cosh \frac{\beta V}{2}-1\right] .
$$

Equation (35) is the result obtained earlier by Kang et al. [15] and it is the surface-tension renormalization term. Therefore, result (33) may be regarded as a generalization of the surface renormalization term including the steric effect.

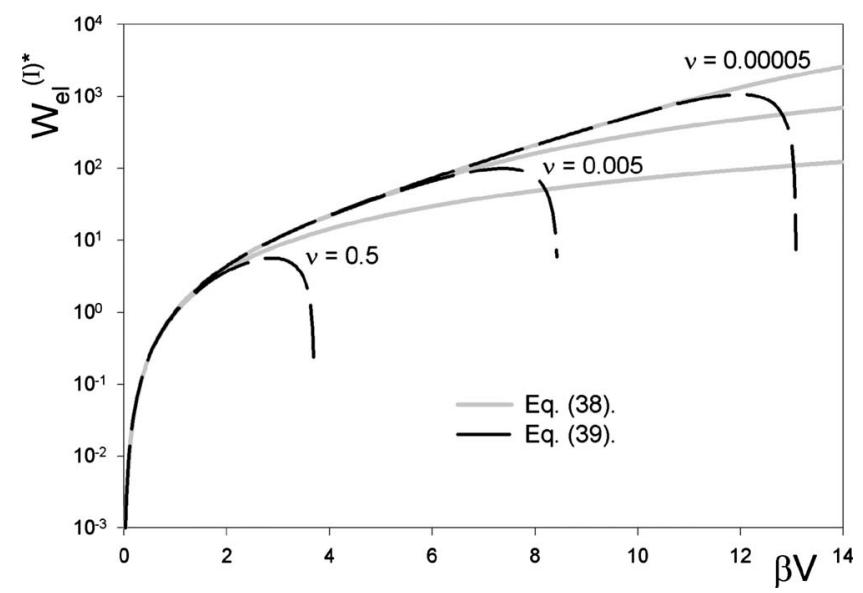

FIG. 4. Dimensionless wetting tension vs $\beta V$ by using Eqs. (38) and (39) for $\nu=0.5,0.005$, and 0.00005 .

The first correction to Eq. (35) to include the steric effect can be done by the approximation for $0<\nu_{j} \ll 1$,

$$
\sqrt{\frac{1}{\nu_{j}} \ln \left[1+\nu_{j}(\cosh \eta-1)\right]} \simeq \sqrt{2} \sinh \frac{\eta}{2}\left(1-\frac{\nu_{j}}{2} \sinh ^{2} \frac{\eta}{2}\right) .
$$

By substituting this approximation to Eq. (33), we have

$$
\begin{aligned}
W_{e l}^{(I)}= & 8 k T\left[\frac{c_{10}}{\kappa_{1}}-\frac{c_{20}}{\kappa_{2}}\right]\left[\cosh \frac{\beta V}{2}-1\right]-\frac{4 k T}{3}\left[\frac{c_{10}}{\kappa_{1}} \nu_{1}-\frac{c_{20}}{\kappa_{2}} \nu_{2}\right] \\
& \times\left[\cosh \frac{\beta V}{2}+2\right]\left[\cosh \frac{\beta V}{2}-1\right]^{2}+O\left(\nu_{j}^{2}\right) .
\end{aligned}
$$

From (37), we can see that, if the surface potential goes to zero, the wetting tension formula reduces to the form without the steric effect [Eq. (35)].

As a special case, let us consider a typical case of an electrolyte droplet (fluid 1) attached on a constant potential surface in an inert gas medium (fluid 2). In this case, $c_{20} / \kappa_{2}=0$ and Eq. (33) reduces to the following result in dimensionless form:

$\left.W_{e l}^{(I) *}=\frac{W_{e l}^{(I)}}{\left.\left[c_{10} k T\right)\right] / \kappa_{1}}=2 \sqrt{2} \int_{0}^{\beta V} \sqrt{\frac{1}{\nu_{1}} \ln \left[1+\nu_{1}(\cosh \eta-1)\right.}\right] d \eta$

and when $0<\nu_{1} \ll 1$ we have

$$
\begin{aligned}
W_{e l}^{(I) *}= & 8\left[\cosh \frac{\beta V}{2}-1\right] \\
& \times\left[1-\frac{\nu_{1}}{6}\left(\cosh \frac{\beta V}{2}+2\right)\left(\cosh \frac{\beta V}{2}-1\right)+O\left(\nu_{1}^{2}\right)\right] .
\end{aligned}
$$

The results of Eqs. (38) and (39) are shown in Fig. 4.

From above, we can see also that the steric effect up to the first order always reduces the wetting tension because the factor $[\cosh (\beta V / 2)+2][\cosh (\beta V / 2)-1]>0$. Furthermore, the relative reduction increases as the magnitude of the sur- 


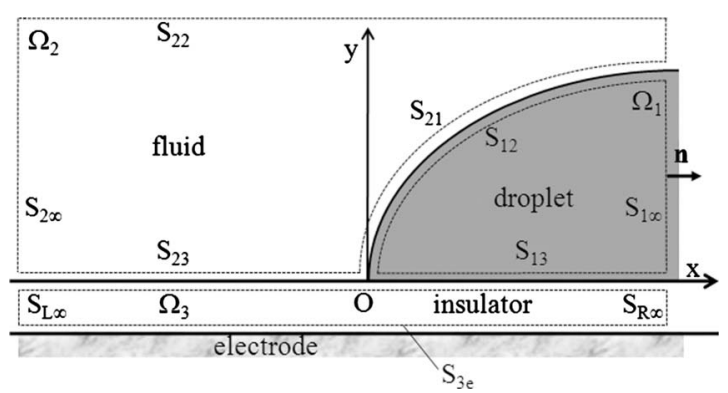

FIG. 5. Control surfaces for the analysis of electrowetting problem.

face potential increases. This prediction of the tendency matches perfectly with our intuitive expectation of the steric effect.

\section{Constant charge problem (case II)}

The difference of the constant charge problem from the previous constant potential problem is that $\mathbf{E} \cdot \mathbf{e}_{x} \neq 0$ along the substrate surface. Therefore, we have

$$
f_{1 x}^{(I I)}=-\int_{S_{1 \infty}}\left[\pi+\frac{1}{2} \epsilon_{1} E^{2}\right] d s+\int_{S_{13}} \epsilon_{1}(\mathbf{n} \cdot \mathbf{E}) \mathbf{E} \cdot \mathbf{e}_{x} d s .
$$

As shown in Kang et al. [15], $\mathbf{E}=-\boldsymbol{\nabla} \phi$ and $\left.\epsilon_{1} \mathbf{E} \cdot \mathbf{n}\right|_{S_{13}}=-\sigma_{1}$, where $\sigma_{1}$ is the surface charge density at the substrate surface. So,

$$
\begin{aligned}
\int_{S_{13}} \epsilon_{1}(\mathbf{n} \cdot \mathbf{E}) \mathbf{E} \cdot \mathbf{e}_{x} d s & =\sigma_{1} \int_{0}^{\infty} \frac{\partial \phi}{\partial x} d x \\
& =\sigma_{1}\left[\phi_{1}(\infty, 0)-\phi_{1}(0,0)\right] .
\end{aligned}
$$

Since all other terms are the same as the constant potential case, we have

$$
\begin{aligned}
W_{e l}^{(I I)}= & \left.2 \sqrt{2}\left[\frac{c_{10} k T}{\kappa_{1}}\right] \int_{0}^{\beta \phi_{1 \infty}} \sqrt{\frac{1}{\nu_{1}} \ln \left[1+\nu_{1}(\cosh \eta-1)\right.}\right] d \eta \\
& \left.-2 \sqrt{2}\left[\frac{c_{20} k T}{\kappa_{2}}\right] \int_{0}^{\beta \phi_{2 \infty}} \sqrt{\frac{1}{\nu_{2}} \ln \left[1+\nu_{2}(\cosh \eta-1)\right.}\right] d \eta \\
& +\left[\sigma_{1}-\sigma_{2}\right] \phi_{0}-\left[\sigma_{1} \phi_{1 \infty}-\sigma_{2} \phi_{2 \infty}\right]
\end{aligned}
$$

where $\phi_{0}=\phi(0,0)$.

\section{Electrowetting on dielectrics (case III)}

For computation of wetting tension, we consider a control system shown in Fig. 5. As in previous subsections, we calculate the $x$-component of the surface force per unit depth as

$$
\begin{aligned}
W_{x}^{(I I I)} & =-f_{x}^{(I I I)} \\
& =-\mathbf{e}_{x} \cdot \int_{S_{1 \infty}+S_{13}} \mathbf{T} \cdot \mathbf{n} d s-\mathbf{e}_{x} \cdot \int_{S_{2 \infty}+S_{23}+S_{22}} \mathbf{T} \cdot \mathbf{n} d s .
\end{aligned}
$$

The above can be written as

$$
\begin{aligned}
W_{e l}^{(I I I)}= & -\mathbf{e}_{x} \cdot \int_{S_{1 \infty}} \mathbf{T} \cdot \mathbf{n} d s-\mathbf{e}_{x} \cdot \int_{S_{2 \infty}} \mathbf{T} \cdot \mathbf{n} d s \\
& -\int_{S_{13}+S_{23}} \epsilon\left(\mathbf{E} \cdot \mathbf{e}_{x}\right) \mathbf{E} \cdot \mathbf{n} d s .
\end{aligned}
$$

The first two terms are obtained as in the previous subsections. The last term can be easily calculated according to the method in Kang et al. [15] of which the idea can be summarized as follows.

The integrals over the surfaces $S_{13}$ and $S_{23}$ can be transformed to the integrals over the surface $S_{31}$ and $S_{32}$ by using the matching conditions of the normal and tangential components of electric field. Under the assumption that no charge is adsorbed at the fluid-solid interface, we have

$$
\left.\boldsymbol{\epsilon} \cdot \mathbf{n}\right|_{S_{13}, S_{23}}=-\left.\boldsymbol{\epsilon} \mathbf{E} \cdot \mathbf{n}\right|_{S_{31}, S_{32}},\left.\quad \mathbf{E} \cdot \mathbf{e}_{x}\right|_{S_{13}, S_{23}}=\left.\mathbf{E} \cdot \mathbf{e}_{x}\right|_{S_{31}, S_{32}} .
$$

We again use the property of Maxwell stress tensor mentioned previously,

$$
\int_{\Omega_{3}} \boldsymbol{\nabla} \cdot \mathbf{T}^{e} d \Omega=\int_{\Sigma_{3}} \mathbf{n} \cdot \mathbf{T}^{e} d \Sigma=\mathbf{0} .
$$

The final result for the wetting tension is

$$
\begin{aligned}
W_{e l}^{(I I I)}= & \frac{\epsilon_{3}}{2 d}\left[\left(V-\phi_{1 \infty}\right)^{2}-\left(V-\phi_{2 \infty}\right)^{2}\right]+2 \sqrt{2}\left[\frac{c_{10} k T}{\kappa_{1}}\right] \int_{0}^{\beta \phi_{1 \infty}} \\
& \times \sqrt{\frac{1}{\nu_{1}} \ln \left[1+\nu_{1}(\cosh \eta-1)\right] d \eta} \\
& -2 \sqrt{2}\left[\frac{c_{20} k T}{\kappa_{2}}\right] \int_{0}^{\beta \phi_{2 \infty}} \sqrt{\frac{1}{\nu_{2}} \ln \left[1+\nu_{2}(\cosh \eta-1)\right]} d \eta,
\end{aligned}
$$

where $d$ is the thickness of the dielectric layer. If the surrounding fluid is air, then $\phi_{2 \infty}=V$ and $c_{20}=0$ and the above equation reduces to

$$
\begin{aligned}
W_{e l}^{(I I I)}= & \frac{\epsilon_{3}}{2 d}\left(V-\phi_{1 \infty}\right)^{2}+2 \sqrt{2}\left[\frac{c_{10} k T}{\kappa_{1}}\right] \int_{0}^{\beta \phi_{1 \infty}} \\
& \times \sqrt{\frac{1}{\nu_{1}} \ln \left[1+\nu_{1}(\cosh \eta-1)\right]} d \eta .
\end{aligned}
$$

Furthermore, if the droplet is a perfect conductor, $\phi_{1 \infty}=0$. In this case, the Lippmann equation is recovered

$$
W_{e l}^{(I I I)}=\frac{\epsilon_{3}}{2 d} V^{2} .
$$

More detailed discussion on the parameters will be given in Sec. IV.

\section{DISCUSSION}

\section{A. Analysis without steric effect (based on PB equation)}

Here we study the effect of electrolyte concentration on the wetting tension. First, we consider the simple case of 
electrowetting of a liquid droplet inside air with no steric effect. In this case, the wetting tension has the form [15]

$$
W_{e l}^{(I I I)}=\frac{\epsilon_{3}}{2 d}\left(V-\phi_{\infty}\right)^{2}+\frac{8 c_{0} k T}{\kappa_{1}}\left[\cosh \frac{\beta \phi_{\infty}}{2}-1\right] .
$$

In order to see the concentration effect, we need to determine the wetting tension as a function of $c_{0}$. To do that, the matching condition at the solid (dielectric layer)-liquid interface is used with the assumption that there is no adsorbed charge

$$
\left.\epsilon_{3} \phi_{3}^{\prime}\right|_{S_{31}}=\left.\epsilon_{1} \phi_{1}^{\prime}\right|_{S_{13}} \text {. }
$$

As $x \rightarrow \infty$, the change is only in $y$ direction. So, the onedimensional version of Eq. (24) without steric effect is

$$
\frac{d^{2} \phi_{1}}{d y^{2}}=\frac{\kappa_{1}^{2}}{\beta} \sinh \beta \phi_{1} \text {. }
$$

By integrating the above equation, we have [with the notations $\phi_{\infty}=\phi_{1}(\infty, 0)$ and $\left.\phi_{1 \infty}^{\prime}=\phi_{1}^{\prime}(\infty, 0)\right]$

$$
\left[\phi_{1 \infty}^{\prime}\right]^{2}=\frac{4 c_{0} k T}{\epsilon_{1}}\left[\cosh \beta \phi_{\infty}-1\right] .
$$

By substituting Eq. (51) into the above equation, we have a relationship between the bulk concentration and the surface potential

$$
4 c_{0} k T=\left[\frac{\epsilon_{3}^{2}}{\epsilon_{1} d^{2}}\right] \frac{\left(V-\phi_{\infty}\right)^{2}}{\left[\cosh \beta \phi_{\infty}-1\right]} .
$$

From above, the right-hand side is a monotonically decreasing function of $\phi_{\infty}\left(0 \leq \phi_{\infty} \leq V\right)$. So, as $c_{0} \rightarrow 0, \phi_{\infty} \rightarrow V$, and as $c_{0} \rightarrow \infty, \phi_{\infty} \rightarrow 0$.

By substituting Eq. (53) into Eq. (50), we have

$$
\begin{aligned}
W_{e l}^{(I I I)} & =\frac{\epsilon_{3}}{2 d}\left(V-\phi_{\infty}\right)^{2}\left[1+4\left\{\frac{\kappa_{1}^{-1}}{\epsilon_{1}} \frac{\epsilon_{3}}{d}\right\}\left\{\frac{\cosh \left(\beta \phi_{\infty} / 2\right)-1}{\cosh \left(\beta \phi_{\infty}\right)-1}\right\}\right] \\
& =\frac{\epsilon_{3}}{2 d}\left(V-\phi_{\infty}\right)^{2}\left[1+\frac{4}{\gamma}\left\{\frac{\cosh \left(\beta \phi_{\infty} / 2\right)-1}{\cosh \left(\beta \phi_{\infty}\right)-1}\right\}\right],
\end{aligned}
$$

where

$$
\gamma=\left[\frac{\epsilon_{1}}{\kappa_{1}^{-1}}\right]\left[\frac{d}{\epsilon_{3}}\right]=\frac{C_{E D L}}{C_{d}}=\sqrt{\frac{2 \epsilon_{1} c_{0} z^{2} e^{2} d^{2}}{\epsilon_{3}^{2} k T}} .
$$

In Eq. (55), $C_{E D L}$ and $C_{d}$ stand for the capacitances per unit area for the electrical double layer (EDL) and the dielectric layer. Therefore, the dimensionless parameter $\gamma$ has the physical meaning as the ratio of two capacitances of EDL and the dielectric layer. The double layer thickness $\kappa^{-1}$ is inversely proportional to $\sqrt{c_{0}}$. Thus $\gamma \rightarrow 0$ as $c_{0} \rightarrow 0$ and $\gamma \rightarrow \infty$ as $c_{0} \rightarrow \infty$.

Now, let us take a look at the concentration effect. When the concentration is very low, we can see that $W_{e l}^{(I I I)} \rightarrow 0$ from Eq. (50). The first term goes to zero because $\phi_{\infty} \rightarrow V$ and the second term also goes to zero because of the factor of $\sqrt{c_{0}}$ (note that $\kappa$ is proportional to $\sqrt{c_{0}}$ ). In the case of high concentration, $\phi_{\infty} \rightarrow 0$ and $\kappa_{1} \rightarrow \infty$. From Eq. (54), we can see that

$$
W_{e l}^{(I I I)} \rightarrow \frac{\epsilon_{3}}{2 d} V^{2} \quad \text { as } \quad c_{0} \rightarrow \infty .
$$

So, in the high-concentration limit, the electrolyte behaves like a pure conductor.

In order to see the concentration effect more explicitly, let us consider the case of low applied voltage. By substituting the approximation $\cosh \beta \phi_{\infty} \sim 1+\frac{1}{2}\left(\beta \phi_{\infty}\right)^{2}$ to Eq. (53) with $\beta=z e / k T$, we have

$$
\xi \equiv \frac{\phi_{\infty}}{V}=\frac{1}{1+\gamma} .
$$

By substituting Eq. (57) into Eq. (54) for small $\beta \phi_{\infty}$, we have

$$
W_{e l}^{(I I I)}=\frac{\epsilon_{3}}{2 d} V^{2}\left(\frac{\gamma}{\gamma+1}\right) .
$$

From Eq. (58), we can see that the wetting tension is proportional to the square of the applied voltage just like a pure conductor without regard to concentration in the case of small $\beta \phi_{\infty}$. The concentration changes only the factor. Now let us consider a pure water droplet as an example. In this case, $c_{0}=10^{-7} \mathrm{M}$, and $\kappa_{1}^{-1} \sim 10^{-6} \mathrm{~m}=1 \mu \mathrm{m}$. If $\epsilon_{3}=8 \epsilon_{0}$ and $d=10 \mu \mathrm{m}$, then $\gamma \sim 100$ and the water behaves like a pure conductor. On the other hand, if $d=100 \mathrm{~nm}$, then $\gamma \sim 1.0$. In this case, if salt is added, the effect of ion concentration can be noticed. In fact, the recent advances of materials technology make such a thin dielectric layer available [4].

Another point to be made for Eq. (58) is that it can be rewritten as

$$
W_{e l}^{(I I I)}=\frac{\epsilon_{3}}{2 d} V^{2}\left(\frac{\gamma}{\gamma+1}\right)=\frac{1}{2} V^{2} \frac{C_{E D L} C_{d}}{C_{E D L}+C_{d}}=\frac{1}{2} C_{t o t} V^{2},
$$

where $C_{t o t}$ is the total capacitance per unit area that is obtained by

$$
\frac{1}{C_{t o t}}=\frac{1}{C_{E D L}}+\frac{1}{C_{d}} .
$$

The above result tells us that the two serial capacitors model, which has been used by many researchers, is correct in the limit of small applied potential. This fact also manifests the correctness and applicability of the electromechanical approach.

For wider range of applied voltages, we must solve the set of the original equations. Equation (53) can be written in terms of $\gamma$ and $\beta V$ as

$$
\frac{2 \gamma^{2}}{(\beta V)^{2}}=\frac{(1-\xi)^{2}}{\cosh (\beta V \xi)-1} .
$$

We solve the above equation for $\xi$ and substitute it for

$$
W_{e l}^{(I I I) * *}=\frac{W_{e l}^{(I I I)}}{\epsilon_{3} V^{2} / 2 d}=(1-\xi)^{2}\left[1+\frac{4}{\gamma}\left(\frac{\cosh (\beta V \xi / 2)-1}{\cosh (\beta V \xi)-1}\right)\right] .
$$

In the above, the dimensionless wetting tension is defined as the ratio of the obtained wetting tension to the wetting ten- 


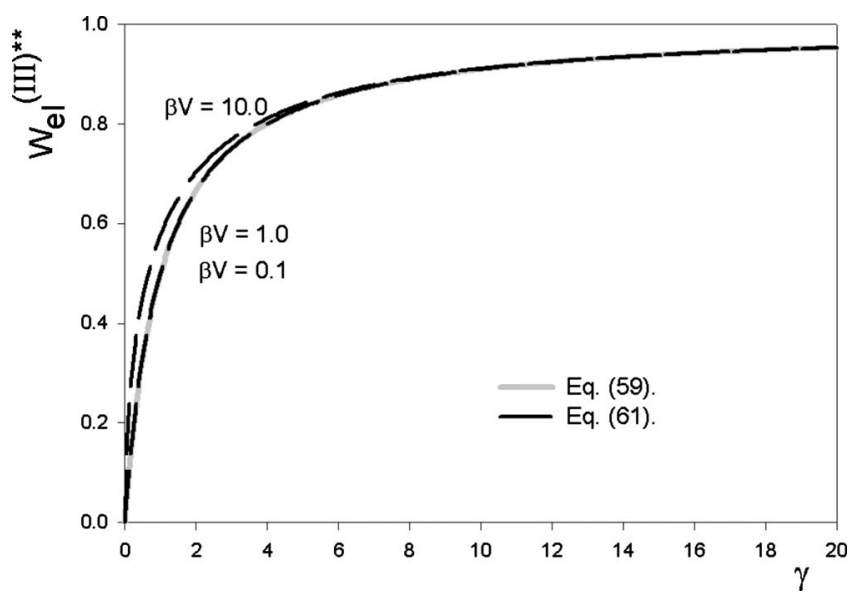

FIG. 6. Effect of electrolyte concentration on the wetting tension when no steric effect is considered $\left(\gamma=\sqrt{2 \epsilon c_{0} z^{2} e^{2} d^{2} / \epsilon_{3}^{2} k T}\right)$.

sion of the ideal case of perfectly conducting fluid. The results are shown in Fig. 6, which shows that the dimensionless wetting tension approaches unity as $\gamma$ increases. Also shown is that for small values of $\gamma$, the dimensionless wetting tension increases as $\beta V$ increases.

\section{B. Analysis with steric effect (based on mPB equation)}

When we have a gas phase as the phase 2, from Eq. (48), we have the formula for the wetting tension

$$
\begin{aligned}
W_{e l}^{(I I I)}= & \frac{\epsilon_{3}}{2 d}\left(V-\phi_{\infty}\right)^{2}+2 \sqrt{2}\left[\frac{c_{0} k T}{\kappa_{1}}\right] \int_{0}^{\beta \phi_{\infty}} \\
& \times \sqrt{\frac{1}{\nu_{1}} \ln \left[1+\nu_{1}(\cosh \eta-1)\right]} d \eta .
\end{aligned}
$$

From the matching conditions [Eq. (51)], we have

$$
\frac{4 c_{0} k T}{\epsilon_{1}} \frac{1}{\nu_{1}} \ln \left[1+\nu_{1}\left(\cosh \beta \phi_{\infty}-1\right)\right]=\frac{\epsilon_{3}^{2}}{\epsilon_{1}^{2}} \frac{\left(V-\phi_{\infty}\right)^{2}}{d^{2}} .
$$

The above condition may be further simplified into the following dimensionless form:

$$
\frac{2 \gamma^{2}}{\nu_{1}} \ln \left[1+\nu_{1}(\cosh \beta V \xi-1)\right]=(\beta V)^{2}(1-\xi)^{2} .
$$

For the given values of $\beta V, \gamma$, and $\nu_{1}$, we solve the above equation to determine $\xi$, which can be substituted into the following equation to have the dimensionless wetting tension as:

$$
\begin{aligned}
W_{e l}^{(I I I) * *}= & \frac{W_{e l}^{(I I I)}}{\epsilon_{3} V^{2} /(2 d)} \\
= & \frac{2 \gamma}{(\beta V)^{2}}\left[\frac{\gamma}{\nu_{1}} \ln \left\{1+\nu_{1}(\cosh (\beta V) \xi-1)\right\}\right. \\
& \left.+\sqrt{2} \int_{0}^{\beta V \xi} \sqrt{\frac{1}{\nu_{1}} \ln \left\{1+\nu_{1}(\cosh \eta-1)\right\}} d \eta\right]
\end{aligned}
$$

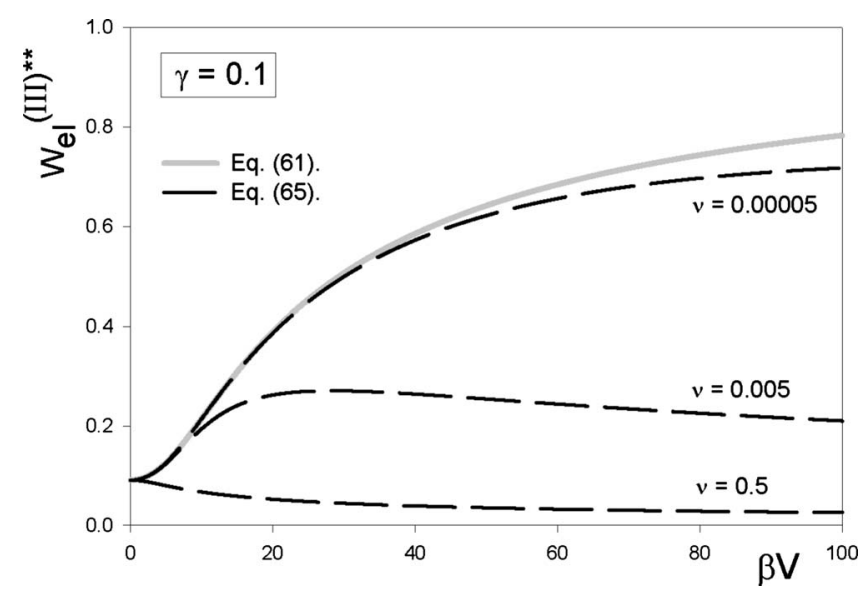

FIG. 7. The dimensionless wetting tension as a function of dimensionless applied voltage $\left(W_{e l}^{(I I I) * *}\right.$ vs $\left.\beta V\right)$ in the case of $\gamma=0.1$.

The dimensionless wetting tension $\left(W_{e l}^{(I I I) * *}\right)$ in terms of dimensionless applied voltage is shown for various values of the steric effect parameter $\left(\nu=\nu_{1}\right)$ in Fig. 7 for the case $\gamma=0.1$. From the figure, we can see that the steric effect decreases the wetting tension significantly compared to the no steric effect case. Even for small $\nu$ value, the effect can be noticed. This tendency gets even more prominent for higher applied voltage (as $\beta V$ increases). Because of the steric effect, the concentration of the counterion near the interface is limited by the steric hindrance. Thus the wetting tension decreases as the hindrance effect increases (as $\nu$ increases). As we have seen before, the parameter $\gamma$ is the ratio of $C_{E D L}$ to $C_{d}$. Thus, when $\gamma$ is small, the effect of EDL is dominant on the total capacitance. Therefore, the hindrance effect is quite noticeable.

In Figs. 8 and 9, similar plots are given for the cases of $\gamma=1$ and $\gamma=10$, respectively. Compared with the case of $\gamma=0.1$, we can see that the steric hindrance effect is less significant as $\gamma$ increases. By the definition, the increase of $\gamma$ means that $C_{E D L} / C_{d}$ is increased and this means the contribution of EDL gets smaller in the contribution to the total capacitance. Therefore, the hindrance effect decreases as $\gamma$ is increased.

In Fig. 10, the dimensionless potential at the liquid-solid interface $\left(\xi=\phi_{\infty} / V\right)$ is shown for the case $\gamma=0.1$. As mentioned earlier, the $\xi$ value can be obtained by solving Eq. (64). For the given applied voltage, the concentration of the counterion decreases due to hindrance effect. This means that the shielding effect is decreased and the potential at the liquid-solid interface increases.

To appreciate a typical size of the steric effect parameter $\nu$, let us consider an example. If $c_{0}=0.1 \mathrm{M}$ and $a=0.3 \mathrm{~nm}=3 \times 10^{-10} \mathrm{~m}, \nu=2 a^{3} c_{0} \sim 3 \times 10^{-3}$. So, by using Figs. 7-9, we can estimate the effect of steric hindrance when the bulk concentration of ions is $c_{0}=0.1 \mathrm{M}$

Here we need to mention a recent work by Monroe et al. [20]. They considered the electrowetting problem of two conducting liquids by adopting the energy method. By modeling the electrolyte system as combination of the inner layer and the electrical double layer, they computed total capacitance. When the magnitude of the applied voltage is in- 


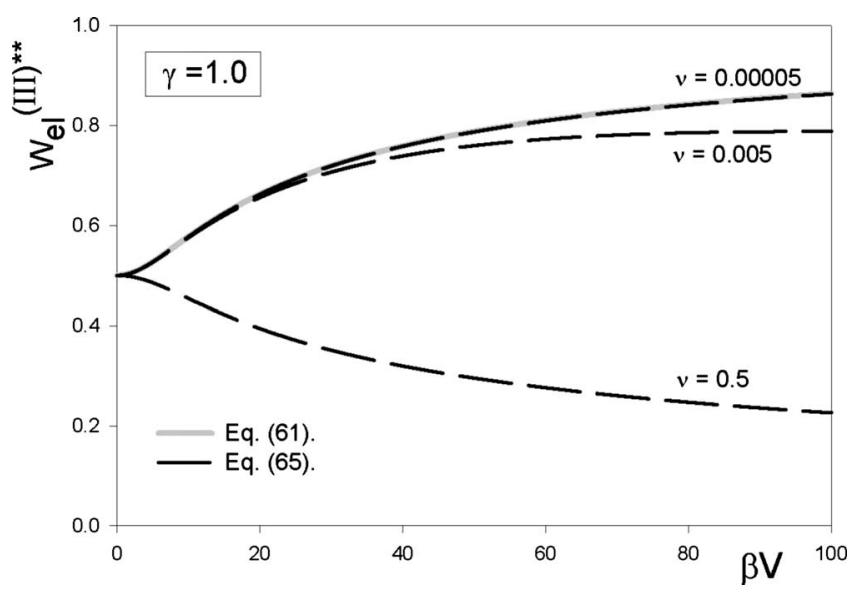

FIG. 8. The dimensionless wetting tension as a function of dimensionless applied voltage $\left(W_{e l}^{(I I I) * *}\right.$ vs $\left.\beta V\right)$ in the case of $\gamma=1$.

creased, the capacitance increases initially and then decreases slowly. The behavior is similar to the curve of $\nu=0.005$ in Fig. 7 of the present work. This point is interesting and provides a motivation of further investigation. It would be interesting if we study the connection between the results obtained by the two different approaches.

\section{CONCLUSION}

In this work, the electromechanical approach is adopted to evaluate the steric effects of ions on the charge-related wetting phenomena. By using the simplified equation of motion under the limit of thin EDL, the complicated problem of integration of electrical stress over the droplet-fluid interface is replaced by the integrations over the straight droplet-solid and fluid-solid interfaces and the boundaries at infinity. In that way, the formulas for the steric effects of the finite-size ions on wetting phenomena are obtained. By analyzing the formulas, we have reached the following conclusions:

(1) the osmotic pressure decreases as the steric effect of ions increases;

(2) for the electrowetting problem with no steric effect, we have identified a very important parameter $\gamma$. That is the

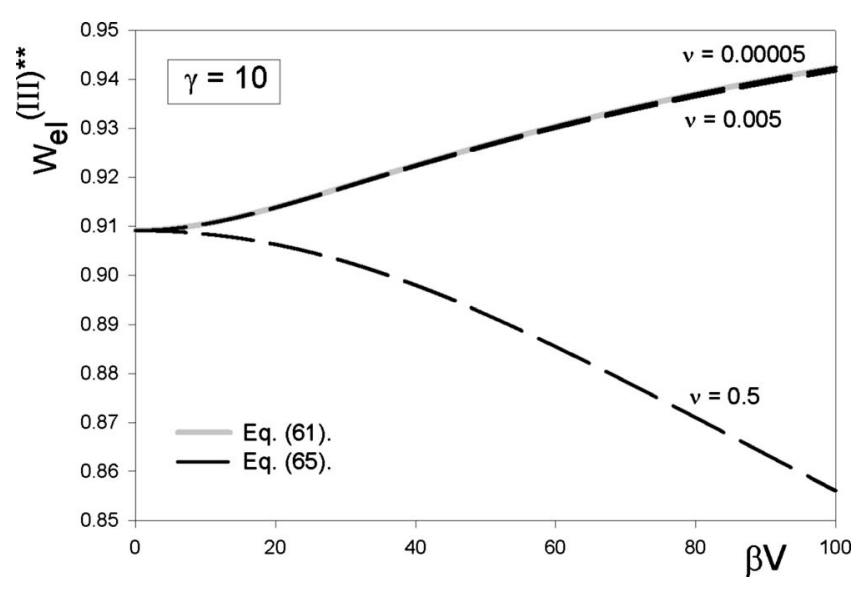

FIG. 9. The dimensionless wetting tension as a function of dimensionless applied voltage $\left(W_{e l}^{(I I I) * *}\right.$ vs $\left.\beta V\right)$ in the case of $\gamma=10$.

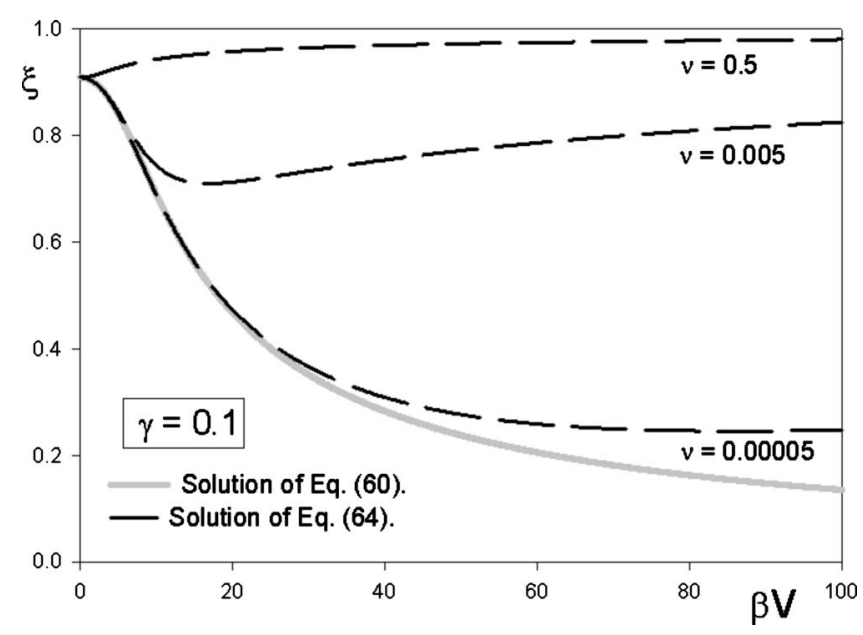

FIG. 10. The ratio of the potential at liquid-solid interface to the applied voltage.

ratio of the capacitance of the electrical double layer to that of the dielectric layer, i.e., $\gamma=C_{E D L} / C_{d}=\left(\epsilon_{1} / \kappa_{1}^{-1}\right)\left(d / \epsilon_{3}\right)$, where $\epsilon_{1}, \epsilon_{3}$ are the electrical permittivities of electrolyte solution and dielectric layer, $\kappa_{1}^{-1}$ the thickness of EDL, and $d$ the dielectric layer thickness. As $\gamma$ increases, the thickness of EDL decreases and the droplet phase behaves like a pure conductor.

(3) When the applied potential is low and there is no steric effect, the formula of the wetting tension derived using the electromechanical approach for the electrowetting problem is reduced to

$$
W_{e l}^{(I I I)}=\frac{1}{2} C_{t o t} V^{2} \quad \text { with } \quad \frac{1}{C_{t o t}}=\frac{1}{C_{E D L}}+\frac{1}{C_{d}} .
$$

This result is exactly consistent with the formula based on the model of serial capacitances, which has been adopted by many previous researchers.

(4) In the case of steric effect, the effects of finite-size ions become more significant as the parameter $\gamma$ decreases. Small $\gamma$ means that $C_{E D L}$ is small compared to $C_{d}$ and EDL has more effect on the overall capacitance as we can see in the above equation. Thus the steric hindrance effect becomes more prominent when $\gamma$ is small. When $\gamma$ is large, the droplet behaves like a pure conductor and the steric effect of finite-size ions becomes negligible.

(5) As the steric effect increases, the shielding effect due to concentrated charges near the liquid-solid interface decreases. Consequently, when the positive potential is applied to the bottom electrode, the potential value at the liquid-solid interface increases.

\section{ACKNOWLEDGMENTS}

This work was supported by the grant from KOSEF. This work was also supported by the BK21 program of Ministry of Education of Korea. The authors greatly acknowledge the financial support. 
[1] F. Mugele and J.-C. Baret, J. Phys.: Condens. Matter 17, R705 (2005).

[2] E. Seyrat and R. A. Hayes, J. Appl. Phys. 90, 1383 (2001).

[3] H. Moon, S. K. Cho, R. L. Garrel, and C. J. Kim, J. Appl. Phys. 92, 4080 (2002).

[4] S. Berry, J. Kedzierski, and B. Abedian, J. Colloid Interface Sci. 303, 517 (2006).

[5] K. Zhou and J. Heikenfeld, Appl. Phys. Lett. 92, 113515 (2008).

[6] M. S. Kilic, M. Z. Bazant, and A. Ajdari, Phys. Rev. E 75, 021502 (2007).

[7] J. J. Bikerman, Philos. Mag. 33, 384 (1942).

[8] I. Borukhov, D. Andelman, and H. Orland, Phys. Rev. Lett. 79, 435 (1997).

[9] I. Borukhov, D. Andelman, and H. Orland, Electrochim. Acta 46, 221 (2000).

[10] M. S. Kilic, M. Z. Bazant, and A. Ajdari, Phys. Rev. E 75,
021503 (2007).

[11] A. A. Kornyshev, J. Phys. Chem. B 111, 5545 (2007).

[12] M. Bazant, M. Kilic, B. Storey, and A. Ajdari, e-print arXiv:0903.4790.

[13] T. B. Jones, Langmuir 18, 4437 (2002).

[14] K. H. Kang, Langmuir 18, 10318 (2002).

[15] K. H. Kang, I. S. Kang, and C. M. Lee, Langmuir 19, 5407 (2003).

[16] J. Buehrle, S. Herminghaus, and F. Mugele, Phys. Rev. Lett. 91, 086101 (2003).

[17] L. D. Landau, E. M. Lifshitz, and L. P. Pitaevskii, Electrodynamics of Continuous Media, 2nd ed. (Elsevier ButterworthHeinemann, Burlington, MA, 1984).

[18] E. Trizac and J.-P. Hansen, Phys. Rev. E 56, 3137 (1997).

[19] R. A. Marcus, J. Chem. Phys. 23, 1057 (1955).

[20] C. W. Monroe, M. Urbakh, and A. A. Kornyshev, J. Electrochem. Soc. 156, P21 (2009). 